\title{
MicroRNA-146a inhibits epithelial mesenchymal transition in non-small cell lung cancer by targeting insulin receptor substrate 2
}

\author{
DONG HO PARK ${ }^{1}$, HYO SUNG JEON ${ }^{2}$, SOO YOUNG LEE ${ }^{3}$, YI YOUNG CHOI ${ }^{4}$, HAE WOO LEE ${ }^{5}$, \\ SEONGKYU YOON ${ }^{6}$, JAE CHEL LEE ${ }^{7}$, YOO SANG YOON ${ }^{8}$, DAE SUNG KIM ${ }^{9}$, MOON JUN NA ${ }^{9}$, \\ SUN JUNG KWON ${ }^{9}$, DONG SUN KIM ${ }^{10}$, JAEKU KANG ${ }^{11}$, JAE YONG PARK ${ }^{12}$ and JI WOONG SON ${ }^{9}$ \\ ${ }^{1}$ Department of Anesthesiology and Pain Medicine, Konyang University Hospital, Daejeon; \\ ${ }^{2}$ The Molecular Diagnostics and Imaging Research Institute, School of Medicine, \\ Kyungpook National University, Daegu; ${ }^{3}$ Myunggok Research Institute for Medical Science, \\ Konyang University, Daejeon; ${ }^{4}$ Department of Biochemistry, School of Medicine, \\ Kyungpook National University; ${ }^{5}$ Clinical Drug Manufacturing Center, \\ Daegu-Gyeongbuk Medical Innovation Foundation, Daegu, Republic of Korea; \\ ${ }^{6}$ Department of Chemical Engineering, University of Massachusetts Lowell, MA, USA; \\ ${ }^{7}$ Department of Oncology, Asan Medical Center, University of Ulsan, College of Medicine, Seoul; \\ Departments of ${ }^{8}$ Thoracic Surgery and ${ }^{9}$ Internal Medicine, Konyang University Hospital, Daejeon; \\ ${ }^{10}$ Department of Anatomy, School of Medicine, Kyungpook National University, Daegu; \\ ${ }^{11}$ Department of Pharmacology, College of Medicine, Konyang University, Daejeon; \\ ${ }^{12}$ Lung Cancer Center, Kyungpook National University Medical Center, Daegu, Republic of Korea
}

Received May 11, 2015; Accepted June 22, 2015

DOI: $10.3892 /$ ijo.2015.3111

\begin{abstract}
During cancer progression, some tumor cells show changes in their plasticity by morphological and phenotypical conversions, as an expression of mesenchymal markers and loss of epithelial markers, collectively referred to as epithelialmesenchymal transition (EMT). EMT has been increasingly recognized as a critical phenomenon in lung cancer progression. The goal of this study was to identify microRNAs involved in lung cancer progression. A microarray and qRT-PCR were performed to investigate the miRNA expression profiles in mesenchymal-like lung cancer cells. The role of miR-146a in lung cancer progression was measured by invasion and migration assays in vitro. Bioinformatics and luciferase report assays were used to identify the target of miR-146a. The expression of
\end{abstract}

Correspondence to: Professor Ji Woong Son, Department of Internal Medicine, Konyang University Hospital, Daejeon, Republic of Korea

E-mail: sk1609@hanmail.net

Professor Jae Yong Park, Lung Cancer Center, Kyungpook National University Medical Center, Daegu, Republic of Korea

E-mail: jaeyong@knu.ac.kr

Key words: lung cancer, microRNA, epithelial mesenchymal transition, drug resistance, invasion
miR-146a was reduced in mesenchymal-like lung cancer cell lines. The overexpression of miR-146a induced a marked reduction of the mesenchymal marker and increase the epithelial marker in lung cancer cell lines. Moreover, the overexpression of miR-146a suppressed lung cancer cell migration and invasion. Co-treatment with miR-146a and gefitinib treatment showed a significant reduction of invasion in the resistant lung cancer cells induced by EMT. The expression of miR-146a was downregulated in advanced lung cancer tissues. Insulin receptor substrate 2 (IRS2), an adaptor protein that modulates normal growth, metabolism, survival, and differentiation, was identified as a target of miR-146a. miR-146a regulated the expression of IRS2 at the mRNA and protein levels. These data demonstrate for the first time that miR-146a suppresses lung cancer progression by repressing IRS2 expression. This provides new insight into the post-transcriptional regulation of lung cancer progression by miRNAs, a potential approach for the treatment of lung cancer.

\section{Introduction}

Lung cancer is the leading cause of cancer-related mortality in the developed world, accounting for 26-29\% of all cancer deaths (1). Non-small cell lung cancer (NSCLC) accounts for $80 \%$ of all lung cancer diagnosis and the majority of these patients are diagnosed at an advanced stage. Therefore, the prognosis of locally advanced NSCLC is poor with a 5-year survival rate of $<10 \%$ (2). Despite surgically complete resec- 
tion, lung cancer frequently relapses with metastasis (3). Understanding the mechanism of cancer progression and metastasis could provide an opportunity to identify novel targets for therapeutic intervention.

The metastatic cascade represents a multi-step process that includes migration, invasion, adhesion, proliferation, angiogenesis and lymphangiogenesis. Invasion and migration are initial steps in the metastatic cascade (4). During cancer progression, some tumor cells show changes in their plasticity by morphologic and phenotypic conversion, as an expression of mesenchymal markers and loss of epithelial markers, collectively referred to as epithelial-mesenchymal transition (EMT), which is measured as expression of mesenchymal markers and loss of epithelial markers (5). EMT is increasingly recognized as a critical phenomenon in lung cancer progression and metastasis (6). EMT has been considered as a general biological switch rendering NSCLC sensitive or insensitive to EGFR inhibition. Increased expression of E-cadherin has been associated with clinical activity of EGFR inhibitors in NSCLC patients. Recent studies showed EMT was observed in EGFR mutant lung cancers with acquired resistance to EGFR TKIs (7).

We investigated the microRNA (miRNA) profiles of mesenchymal-like lung cancer cell lines to evaluate the relationship between EMT and miRNA in NSCLC. miR-146a is downregulated in mesenchymal-like lung cancer cell lines. We previously reported a significant association between the pre-miR-146a rs2910164C > G single nucleotide polymorphism (SNP) and lung cancer risk. The effect of this SNP on lung cancer risk was more pronounced in never smokers (8). Insulin receptor substrate 2 (IRS2) is a predicted target of miR-146a using a combined approach involving computational prediction and a whole genome microarray experiment. IRS adaptor proteins link signaling from upstream activators to multiple downstream effectors which modulate normal growth, metabolism, survival and differentiation (9).

In the present study, we determined the differential expression of miR-146a in lung cancer tissues. We hypothesized that miR-146a plays a role in EMT of lung cancer by directly targeting IRS2. To test this hypothesis, we performed luciferase, cell migration and invasion assays.

\section{Materials and methods}

Cell culture and growth conditions. Lung cancer cells (H226, $\mathrm{H} 358, \mathrm{H} 460$, HCC95, HCC827 and H1299) were maintained in RPMI-1640 medium (Gibco BRL, Rockville, MD, USA) with $10 \%$ fetal bovine serum and antibiotics $(100 \mathrm{U} / \mathrm{ml}$ penicillin and $100 \mathrm{mg} / \mathrm{ml}$ streptomycin).

Patients and tissues samples. Tumor and corresponding normal lung tissue specimens were obtained from 78 Korean patients with NSCLC who underwent curative resection at the Kyungpook National University Hospital. None of the patients had received chemotherapy or radiotherapy prior to surgery. Appropriate Institutional Review Board permission was obtained from the participating hospital, and written informed consent was obtained from all subjects. All of the tumor and macroscopically normal lung tissue samples were obtained at the time of surgery, and were rapidly frozen in liquid nitrogen and stored at $-80^{\circ} \mathrm{C}$ until analysis. Tissue samples were histologically confirmed by hematoxylin-eosin staining.

miRNA microarray and human whole genome expression microarray. Agilent's Human miRNA (Rel 14.0) and Agilent Human GE 4x44K (Agilent Technologies, Santa Clara, CA, USA) were used according to the manufacturer's instructions.

TaqMan miRNA expression assay. qRT-PCR analysis for miRNAs was performed in duplicate with MicroRNA assay kit (Applied Biosystems, Foster City, CA, USA) according to the manufacturer's instructions, and RNU6B was used for normalization.

miRNA mimic, miRNA inhibitor and siRNA transfection. Cells were plated in 6 -well plates at a density of $1.2 \times 10^{5}$ cells/well. The next day, cells were transfected with $30 \mathrm{nM}$ pre-miR miRNA mimic (Ambion, Austin, TX, USA), anti-miR inhibitor (Ambion), premiR miRNA mimic-negative control\#1 (Ambion) and anti-miR negative control\#1 inhibitor (Ambion) with Lipofectamine ${ }^{\mathrm{TM}}$ RNAiMAX (Invitrogen, Carlsbad, CA, USA) according to the manufacturer's instructions. Also, cells were transfected with specific Silencer ${ }^{\circledR}$ Select siRNA for IRS2 (s16487, Ambion) and Silencer ${ }^{\circledR}$ Select Negative Control No. 1 siRNA (Ambion) with Lipofectamine RNAiMAX (Invitrogen).

$q R T-P C R$. Total RNA was isolated with TRIzol solution (Ambion) according to the protocols of the manufacturer. The first strand of cDNA was synthesized using the oligo(dT) primer system (Super-Script III First-Strand Synthesis System; Invitrogen). Aliquots of the reaction mixture were used for the qPCR amplification with the CFX96 system (Bio-Rad Laboratories, Hercules, CA, USA) using iQ SYBR Green Supermix (Bio-Rad Laboratories). PCR was run for 40 cycles of denaturation at $95^{\circ} \mathrm{C}$ for $1 \mathrm{sec}$, annealing at $56^{\circ} \mathrm{C}$ for $15 \mathrm{sec}$, and elongation at $72^{\circ} \mathrm{C}$ for $15 \mathrm{sec}$. Gene expression was quantified by the comparative $\mathrm{CT}$ method, with normalization of CT values to the housekeeping gene $\beta$-actin. After amplification, melting curve analysis was performed to ensure the specificity of the products.

Western blot analysis. Cells were lysed in Pro-Prep protein extraction solution (INtRON Biotechnology, Gyeonnggi-do, Korea) $72 \mathrm{~h}$ after transfection. An equal amount of proteins were resolved on $8 \%$ SDS-PAGE gels. The primary antibodies used for the analysis of mouse anti-E-cadherin $(1: 1,000 ; \mathrm{BD}$, Franklin Lakes, NJ, USA), vimentin (1:1,000; Cell Signaling Technology, Beverly, MA, USA), IRS2 (1:1,000; Cell Signaling) antibody and $\beta$-actin antibodies $(1: 2,000$; Santa Cruz Biotechnology, Santa Cruz, CA, USA).

Combined approach to identify the potential target genes of miR-146a. Putative target genes of identified deregulated miRNAs were detected using two different approaches. In the in silico approach, we used the PicTar, TargetScan, miRanda and RNAhybrid. To decrease the number of false-positive results, only putative target genes predicted by at least two programs, were accepted. In the experimental approach, we next examined the whole genome expression microarray in mesenchymal lung cancer cell lines. The overlap between the 

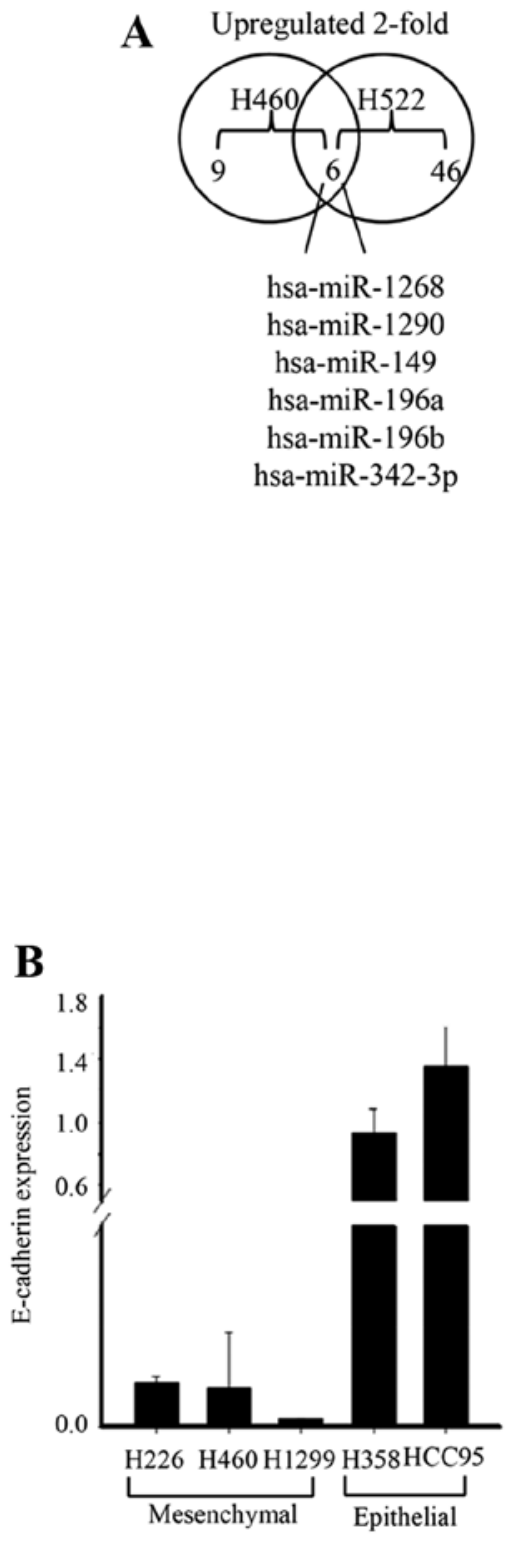
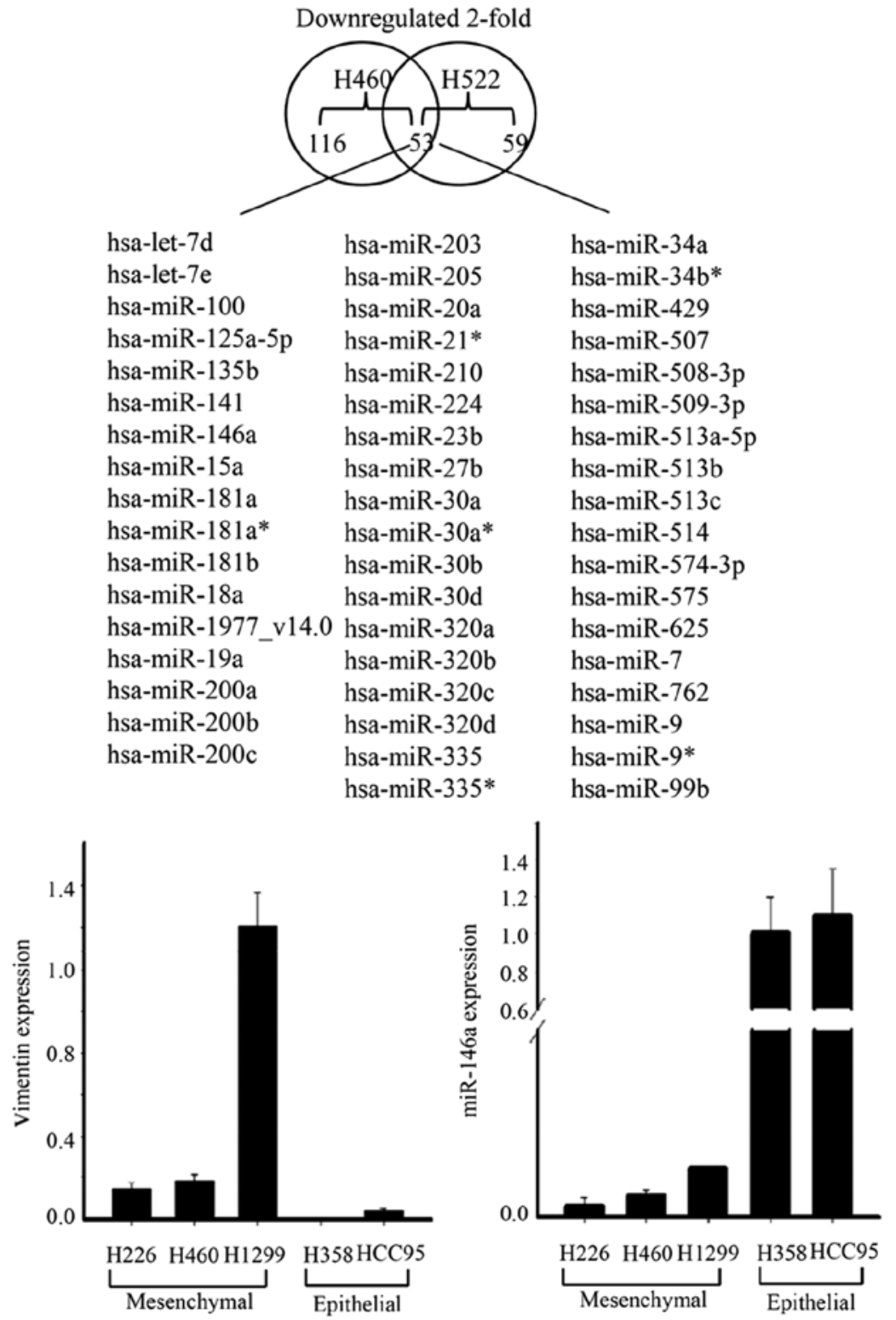

Figure 1. Evaluation of the miRNA expression profiles in mesenchymal-like lung cancer cells. (A) 59 miRNAs significant deregulated miRNAs in mesenchymal-like lung cancer cell lines. (B) miR-146a was downregulated in mesenchymal-like lung cancer cells.

list of mRNA transcripts from the microarray experiments and the putative targets of miR-146a miRNA from in silico approach targets provided the potential target genes regulated by miR-146a.

Luciferase reporter assays. To verify that miR-146a can regulate IRS2 gene directly, we generated a Renilla luciferase reporter plasmid cloned downstream to a segment of the IRS2 3'-UTR containing the putative miR-146a binding sequences, which predicted two binding sites, at position 5158-5186 bp of IRS2 3'-UTR, using the RNAhybrid 2.2 program. The constructs were then co-transfected into $293 \mathrm{~T}$ cells with miR-146a mimic, or mimic-negative control, and Renilla luciferase activity was measured $48 \mathrm{~h}$ later.

Invasion and migration assays. The invasion assay was performed in triplicate using 48-well microchemotaxis chambers (Neuro. Probe, Inc., Gaithersburg, MD, USA) with $8-\mu \mathrm{m}$ pore membranes (Neuro. Probe) pre-coated with $10 \mu \mathrm{g} / \mathrm{ml}$ Matrigel (BD Bioscience). The cells $\left(1 \times 10^{4}\right)$ in $50 \mu \mathrm{l}$ of serum-free medium were placed in the upper chamber, and the lower chamber was filled with $26-27 \mu$ l of medium with $10 \% \mathrm{FBS}$. After incubation for $24 \mathrm{~h}$ at $37^{\circ} \mathrm{C}$, the cells that migrated to the lower surface of the membranes were stained with a Diff-Quick kit and then counted under a microscope. The migration assay was done using the same procedure with membranes that were coated with $5 \mu \mathrm{g} / \mathrm{ml}$ collagen IV (Trevigen, Gaithersbug, MD, USA).

EGFR-tyrosine kinase inhibitor (EGFR-TKI) treatment. The cells were treated with Gefitinib $0.01 \mathrm{M}$ after transfection. The media was changed every $24 \mathrm{~h}$.

Statistical analyses. The statistical differences between groups were analyzed using Student's t-test when two groups were compared. To analyze the drug interaction for synergy, a 
A

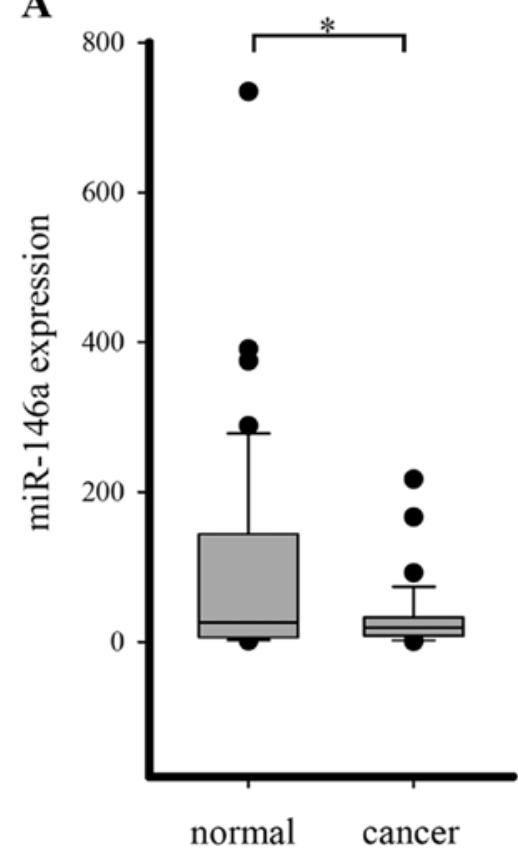

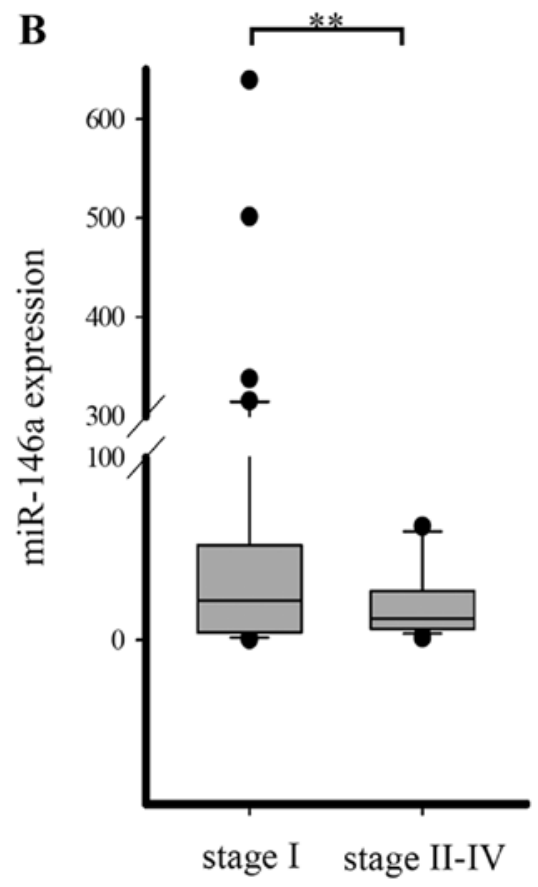

Figure 2. The expression of the miR-146a in lung cancer samples. (A) miR-146a expression in lung cancer and normal lung tissues prepared from the same patients. (B) miR-146a expression in lung cancer tissues according to stages ( $\mathrm{P}=0.231$; $\left.{ }^{* *} \mathrm{P}=0.044\right)$.

one-way classification ANOVA was used. All of the statistical analyses were performed with SPSS 21.0 (SPSS, Chicago, IL, USA). The differences were considered to be statistically significant at $\mathrm{P}<0.05$.

\section{Results}

Identification of differentially expressed microRNAs in mesenchymal lung cancer. We first searched for the microRNAs that showed differential expression between epithelial (H358) and mesenchymal-like lung cancer cell lines (H460, H522) using miRNA microarray (10). We identified 59 miRNAs that were differentially ( $\geq 2$ - to $\leq 0.5$-fold) expressed in both the mesenchymal-like lung cancer cell lines. Among the 59 differentially expressed miRNAs, six were downregulated and 53 miRNAs were underexpressed in mesenchymal-like lung cancer cell lines. To validate the microarray results, the expression of miR-146a was determined using TaqMan miRNA expression assay. Consistent with the results of the microarray analysis, miR-146a was downregulated in the mesenchymal-like lung cancer cell lines (Fig. 1).

Quantitative analysis of miR-146a expression in human lung cancer. A TaqMan miRNA expression assay was applied to detect the miRNA-146a expression in 78 pairs of lung cancer tissue samples and matched normal lung tissue samples. miR-146a was downregulated in lung cancer tissues compared with normal lung, but there was no statistical significance. However, when patients were categorized by lung cancer stages, the expression of miR-146a was significantly downregulated in advanced pathological stages (Fig. 2).

miR-146a targets IRS2. To further analyze the targets of the differentially expressed miRNAs in the mesenchymal-like lung cancer cell lines, we generated mRNA microarray expression profiles in mesenchymal-like lung cancer cell lines (data not shown). The genes changed by 2 -fold matched with putative target genes in the in silico approach were selected as candidates. Several miRNAs overlapped between the list of mRNA transcripts from the microarray experiments and the putative targets of miR-146a from in silico approach. IRS2 was selected as a candidate target of miR-146a through a literature search. To verify that miR-146a regulates the IRS2 gene directly, we generated a Renilla luciferase reporter plasmid cloned downstream to a segment of the IRS2 3-UTR containing the putative miR-146a sequence. Renilla activity was significantly lower by the miR-146a mimic as compared with negative miRNA. To assess whether or not miR-146a has a functional role in the downregulation of endogenous IRS2 expression, we performed qRT-PCR and western blot analysis in H1299 and H460 cells after transfection with negative control or miR-146a mimic. When miR-146a was overexpressed, IRS2 mRNA and protein was diminished compared with the control group (Fig. 3).

miR-146a inhibits lung cancer cell migration and invasion in vitro. To determine if overexpression of miR-146a has an effect on lung cancer progression, we performed invasion and migration assays in a lung cancer cell line. Cells transfected with miR-146a mimic displayed significant reduction of invasion and migration to different degree in the H460 and H1299 cell lines. To confirm that the effect of miR-146a in lung cancer cells occurred through the IRS2 repression, IRS2 siRNA was used to transfect H460 and H1299 cells. IRS2 siRNA decreased lung cancer cell invasion (Fig. 4).

miR-146a overexpression or knockdown of IRS2 regulates EMT-related genes. To elucidate the mechanism underlying 

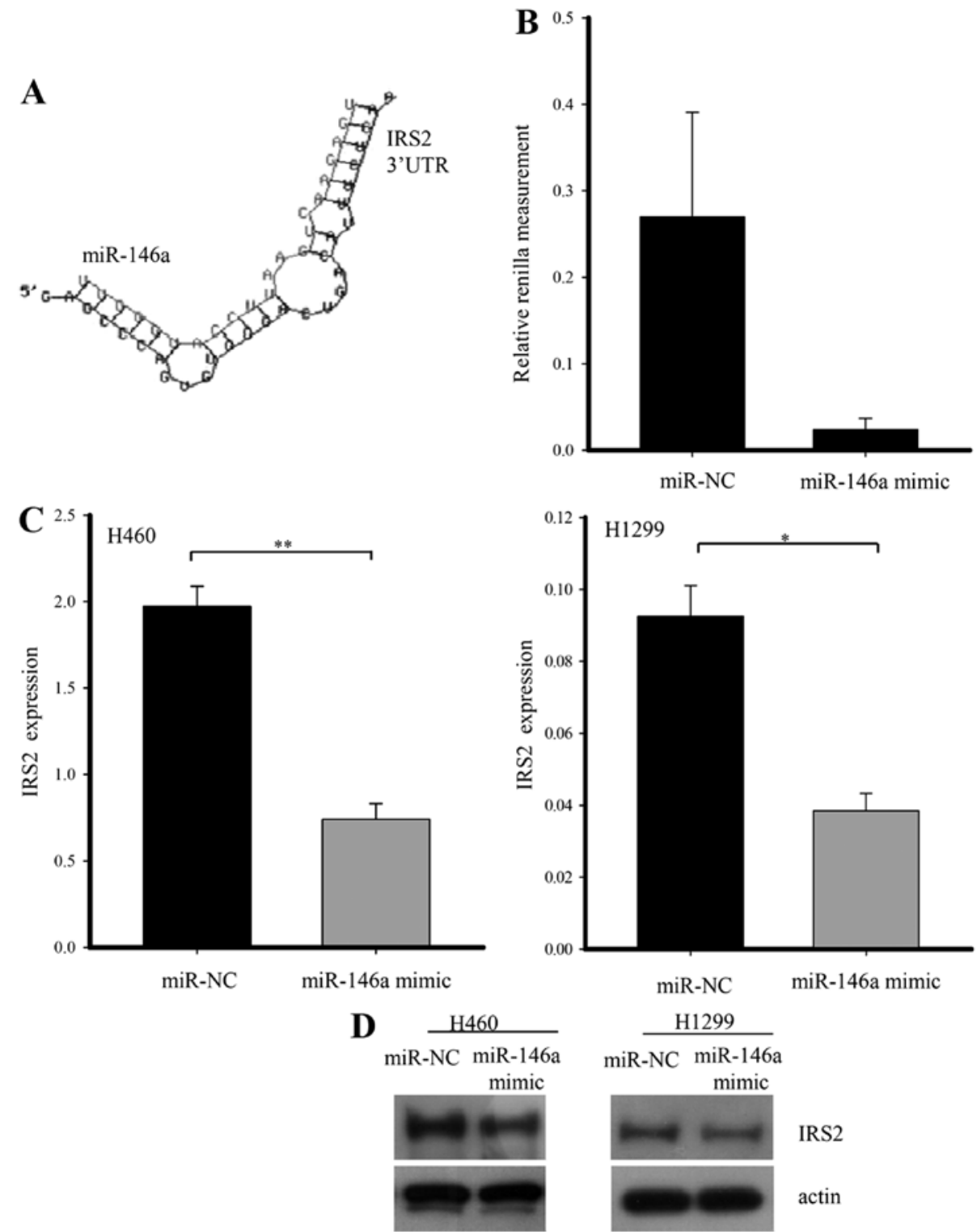

Figure 3. IRS is a direct target gene of miR-146a. (A) The schematic diagram of miR-146a binding sites in the 3 '-untranslated regions (UTR) of IRS2. (B) The signal from a luciferase reporter. (C) The effect of miR-146a mimic on IRS2 mRNA expression in lung cancer cells. (D) The effect of miR-146a mimic on IRS2 protein expression in lung cancer cells. The presented data depict the means $\pm \mathrm{SD}$ of three independent experiments. ${ }^{* *} \mathrm{P}<0.01$ and ${ }^{*} \mathrm{P}<0.05$ as compared to the control.

impaired invasion and migration capacity of miR-146a, we evaluated the expression of an epithelial marker (E-cadherin) and mesenchymal marker (vimentin). The E-cadherin was upregulated in lung cancer cell lines after transfection with the 146a mimic. Ectopic expression of miR-146a was sufficient to reduce expression of vimentin. Conversely, anti-miR-146a induced downregulation of an epithelial marker in HCC95 cells. On the other hand, anti-miR-146a was not sufficient to increase the mesenchymal marker. To confirm that inhibition of EMT by miR-146a in lung cancer occurred through the IRS2 repression, we transfected IRS2 siRNA into lung cancer cells. IRS2 siRNA changed the expression of the epithelial marker and the mesenchymal marker, consistent with the effect of miR-146a (Fig. 5). line to gefitinib treatment. To investigate the role of miR-146a in the chemoresistance induced by EMT, we performed invasion assays in HCC827/CLR cells with acquired resistance to EFGR-TKIs induced by EMT (11). miR-146a mimic markedly decreased invasion of HCC827/CLR cells. Furthermore, combined treatment with miR-146a mimic and gefitinib in HCC827/CLR cells showed a significant reduction of invasion compared to mono-treatment with EGFR-TKI (Fig. 6).

\section{Discussion}

Epithelial and mesenchymal cells are basic tissue phenotypes. The epithelial cells that line the surface of a tissue or organ are attached to the basement membrane, establishing an apical-basal axis of polarity, and communicating with each other through the gap junction, generally not exhibiting a regimented structure or tight intracellular adhesion $(12,13)$. Mesenchymal cells form structures that are irregular in shape 

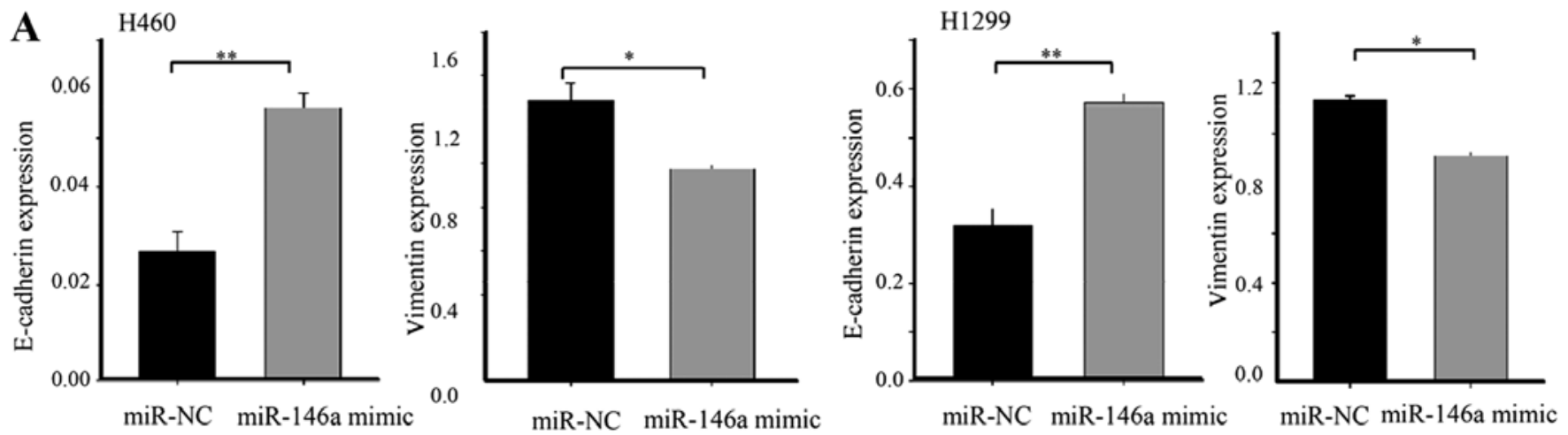

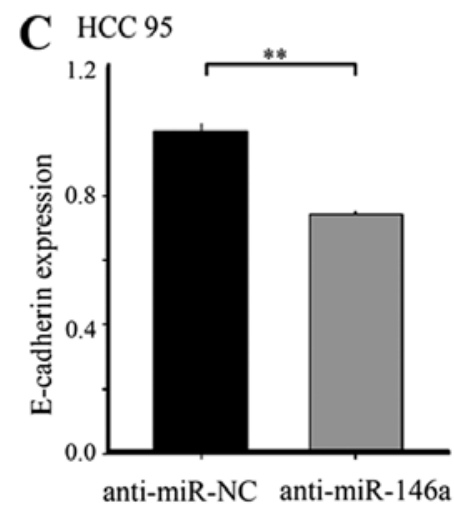

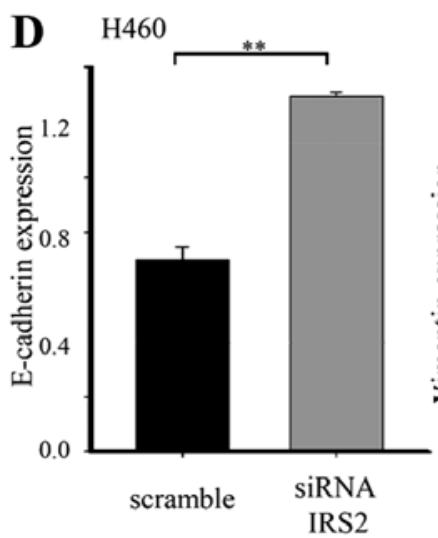
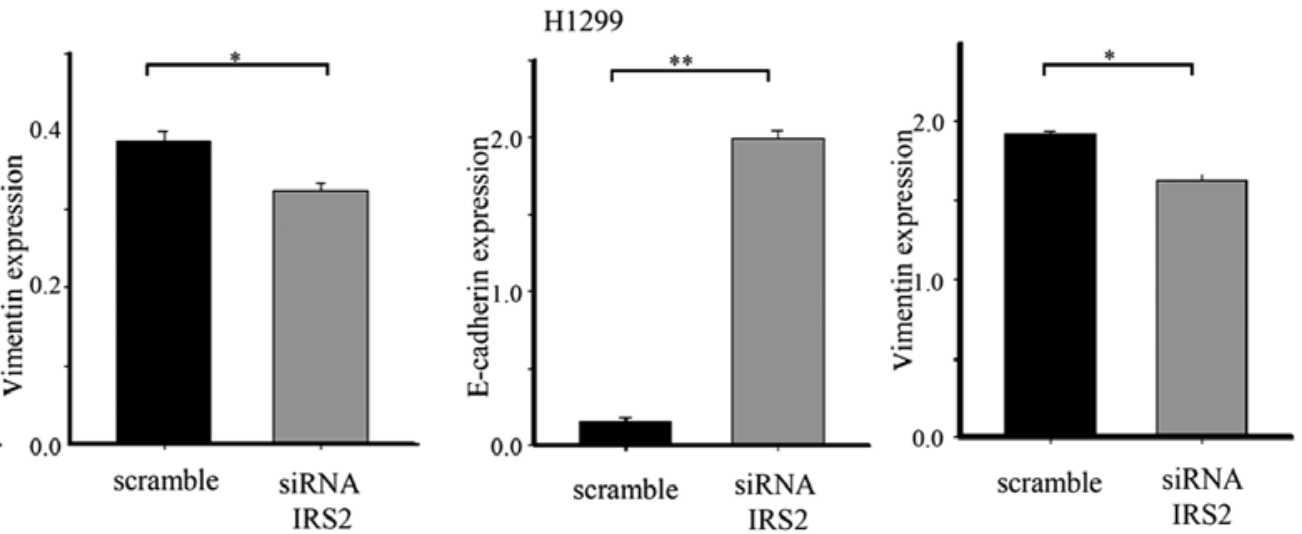

Figure 4. The effects of miR-146a mimic and siRNA IRS2 on EMT related gene expression. (A) The effect of miR-146a mimic on epithelial and mesenchymal specific gene mRNA expression. (B) The effect of miR-146a mimic on epithelial and mesenchymal specific gene protein expression. (C) The effect of antimiR-146a on epithelial and mesenchymal specific gene mRNA expression. (D) The effect of siRNA IRS2 on epithelial and mesenchymal specific gene mRNA expression. The presented data depict the means \pm SD of three independent experiments. ${ }^{* *} \mathrm{P}<0.01$ and ${ }^{*} \mathrm{P}<0.05$ as compared to the control.

and not uniform in composition or density. Adhesion between mesenchymal cells is less strong than in their epithelial counterparts, allowing for increased migratory capacity (14). EMT is a biologic process that allows a polarized epithelial cell to convert into a mesenchymal cell. EMT has an important role in the development of many tissues during embryogenesis, but similar cell changes are recapitulated during pathological processes, such as fibrosis and cancer (15). EMT is a crucial mechanism for cancer drug resistance, progression and metastasis in cancer (16). Recent studies suggest that EMT may occur during lung cancer development. The loss of E-cadherin expression is a hallmark of EMT (17). In some studies, the loss of E-cadherin function also correlates with poor patient prognosis in lung cancers $(18,19)$. The expression of HIF-1 $\alpha$, TWIST1 and Snail, which are regulators of EMT, correlate with survival and relapse in NSCLC (20). L1CAM and SLUG are expressed in the tumor stroma of lung cancer specimens while membranous E-cadherin is expressed in the central regions of the tumor. These findings demonstrate that tumor cells at invasive front have mesenchymal features in a pathologic section $(6,21)$. Serial biopsies demonstrate the transformation from epithelial to mesenchymal cells from the same lung cancer patients. Chung et al (11) reported the morphology of the metastatic tumor was quite different from that of the original lung cancer, which showed mixed acinar and bronchioloalveolar features. Metastasis from lung cancer exhibited spindle-shaped cells with the loss of epithelial polarity. EMT has a role in determining sensitivity to EGFR-TKI or cytotoxic drugs $(22,23)$. Accumulating evidence suggests that EMT is directly implicated in lung cancer. 
A

$\mathrm{H} 460$

miR-NC miR-146a mimic
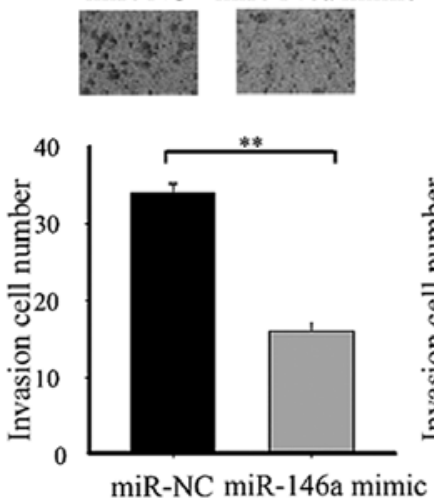

B

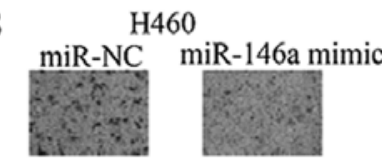

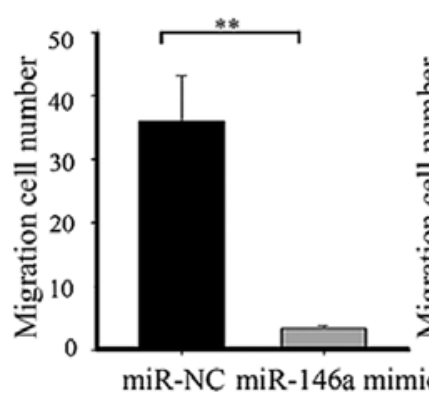

C
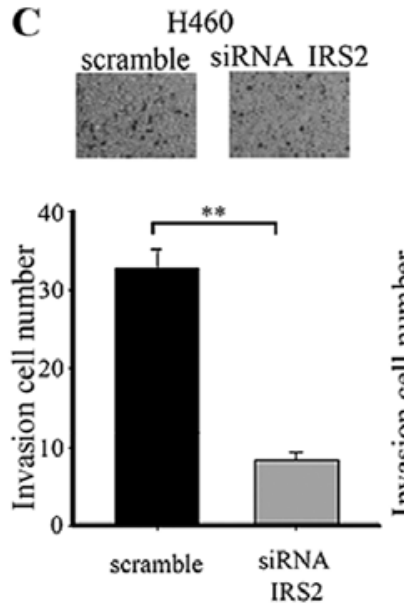

H1299

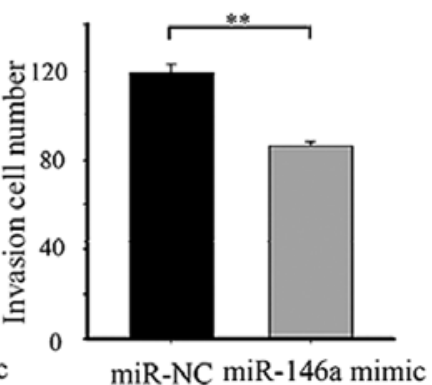

H1299
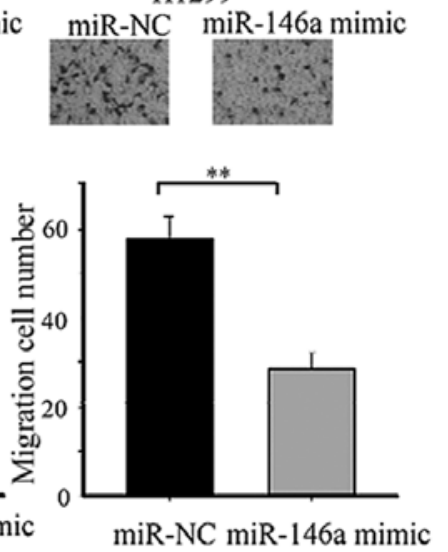

H1299 scramble siRNA IRS2
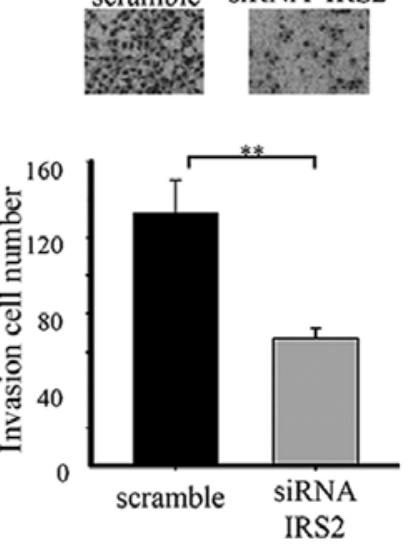
miR-NC miR-146a mimic

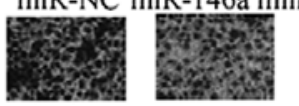

Figure 5. miR-146a mimic inhibits tumor cell invasion and migration in vitro. (A) Microchemotaxis chamber invasion assay of $\mathrm{H} 460$ and $\mathrm{H} 1299$ cells transfected with miR-NC, miR-146a mimic. (B) Microchemotaxis chamber migration assay of $\mathrm{H} 460$ and $\mathrm{H} 1299$ cells transfected with miR-NC, miR-146a mimic. (C) Microchemotaxis chamber invasion assay of H460 and H1299 cells transfected with scramble, siRNA IRS2. The presented data depict the means \pm SD of three independent experiments. ${ }^{* *} \mathrm{P}<0.01$ and ${ }^{*} \mathrm{P}<0.05$ as compared to the control.

miRNAs are non-coding, single-stranded RNAs that repress gene expression by interacting with messenger RNA (mRNA) by inhibiting mRNA translation or by inducing mRNA cleavage. Over 1,400 mature miRNAs have been identified in humans, mostly clustered within the introns of protein coding genes, and targeting roughly $70 \%$ of all genes

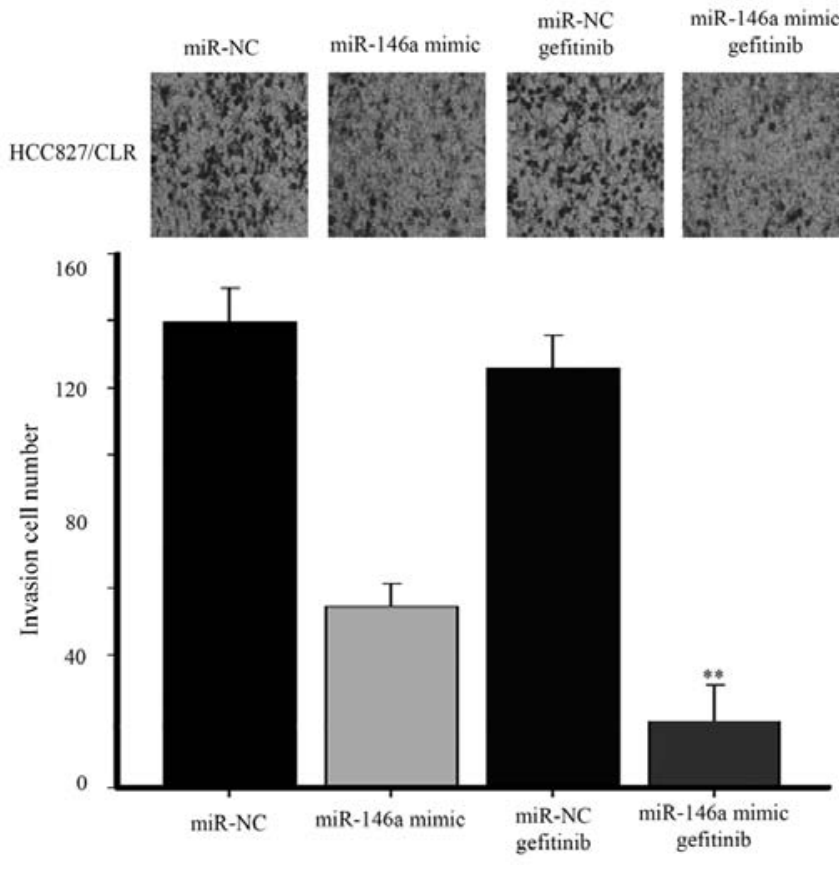

Figure 6. The effect of miR-146a in resistant lung cancer line to EGFR TKIs. The presented data depict the means \pm SD of three independent experiments. ${ }^{* *} \mathrm{P}<0.01$ as compared to the control.

(24). The first evidence that miRNAs can act as regulators of EMT was reported in 2008. The miR-200 family has a role in the control of the epithelial/mesenchymal phenotype of cancer cells (25-27). Loss of cellular miR-200 results in a more mesenchymal-like, highly motile and aggressive phenotype of lung cancer cells (28). Decreased miR-193a-3p/5p expression was significantly associated with lymph node metastasis in lung cancer. The overexpression of miR-193a-3p/5p can inhibit NSCLC cell migration, invasion, and EMT in vitro and lung metastasis formation in vivo. ERBB4 and S6K2 are the direct targets of miR-193a-3p and that PIK3R3 and mTOR were the direct targets of miR-193a-5p in NSCLC (29). Many secreted polypeptides are implicated in the EMT process and their corresponding intracellular transduction pathways form highly interconnected networks. Transforming growth factor- $\beta$, Wnt, Notch, and growth factors acting through tyrosine kinase receptors induce EMT (30). Along with the identification of more signatures of EMT, miRNAs will be have a role as regulators of EMT in lung cancer.

Aberrant expression of miR-146a is involved in progression of various types of cancers (31-33). Chen et al (34) reported the relative miR-146a expression was significantly lower in NSCLC tissues than in the normal lung tissues. The expression of miR-146a was significantly lower in cases with advanced clinical stages and in the cases with metastasis. Overall survival of patients with high miR-146a expression was longer than that of patients with low expression. In their experiments, miR-146a suppressed cell growth and migratory capacity though inhibited EGFR and NF- $\kappa \mathrm{B}$ signaling. In this study, we discovered that IRS2 was a novel direct target of miRNA-146a. IRS2 is a member of insulin receptor substrate (IRS) proteins, an adaptor molecule that integrate extracellular signals from insulin and other ligands to intracellular effec- 
tors such as phosphoinositide 3-kinase and mitogen activated protein kinase. IRS2 is expressed in almost all mammalian tissues and cells. E-cadherin is a key regulator of epithelial cell structure and function, and a loss of E-cadherin is recognized as one of the first steps in the sequence of events in EMT. siRNA-mediated reduction in IRS2 expression significantly increases basal levels of E-cadherin mRNA and protein (35). Wnt signaling plays a pivotal role in cell proliferation, tissue homeostasis, and tumorigenesis. Dishevelled (Dvl) is a central node of Wnt signaling. IRS $1 / 2$ promotes $W n t / \beta$-catenin signaling by stabilizing Dvl2. Overexpression of IRS1/2 increases the protein level of Dvl2 and promotes canonical Wnt signaling, whereas depletion of IRS1/2 reduces the level of Dvl2 and attenuates Wnt/ $\beta$-catenin signaling. IRS1/2 promote the induction of EMT and cell proliferation in response to Wnt stimulation, whereas depletion of Dvl2 impaired the IRS1/2-mediated EMT and cell growth (36). EGFR signaling activated by hypoxia also induces EMT (37). Interestingly, EGF increased IRS-2 promoter activity, while repression of the induction of IRS-2 levels abolishes the EGF enhancement of cell motility (38). Overexpression of IRS1 or IRS2 in the mammary gland showed progressive mammary hyperplasia, tumorigenesis, and metastasis $(9,39,40)$. IRS1 plays a central role in cancer cell proliferation, while IRS2 is associated with cancer cell motility and metastasis. The elimination of IRS1/2 results in long-term inhibition of IGF-IR signaling and powerful inhibition of tumor cell growth (41). This evidence suggested that IRS2 is a major adaptor molecule that links extracellular signals and their corresponding intracellular transduction pathways in EMT.

In this study, we observed substantial IRS2 suppression by miR-146a at the mRNA and protein levels in lung cancer. IRS2 was directly regulated by miR-146a through binding of the 3'-UTR. miR-146a was downregulated in advanced pathological stages and suppressed lung cancer invasion and migration by repressing IRS2 expression. Ectopic expression of miR-146a caused upregulation of E-cadherin in lung cancer cell lines and reduced their motility. Conversely, inhibition of miR-146a reduced E-cadherin expression. miR-146a enhanced the sensitivity of HCC827/CLR to gefitinib. Our data identify miR-146a as a powerful marker for determining the epithelial phenotype of lung cancer cells.

\section{Acknowledgements}

This study was supported by the National Research Foundation of Korea Grant funded by the Korean Government (NRF2012R1A1A4A01005638) and by Konyang University Myunggok Research Fund, 2013.

\section{References}

1. Jemal A, Siegel R, Xu J and Ward E: Cancer statistics, 2010. CA Cancer J Clin 60: 277-300, 2010.

2. Yang P: Epidemiology of lung cancer prognosis: Quantity and quality of life. In: Cancer Epidemiology. Verma M (ed). Humana Press, pp469-486, 2009.

3. Detterbeck FC: Lobectomy versus limited resection in T1N0 lung cancer. Ann Thorac Surg 96: 742-744, 2013.

4. van Zijl F, Krupitza G and Mikulits W: Initial steps of metastasis: Cell invasion and endothelial transmigration. Mutat Res 728: 23-34, 2011.
5. Shih J-Y and Yang P-C: The EMT regulator slug and lung carcinogenesis. Carcinogenesis 32: 1299-1304, 2011.

6. Sato M, Shames DS and Hasegawa Y: Emerging evidence of epithelial-to-mesenchymal transition in lung carcinogenesis. Respirology 17: 1048-1059, 2012.

7. Lin L and Bivona TG: Mechanisms of resistance to epidermal growth factor receptor inhibitors and novel therapeutic strategies to overcome resistance in NSCLC patients. Chemother Res Pract 2012: 817297, 2012.

8. Jeon H-S, Lee YH, Lee SY, Jang JA, Choi YY, Yoo SS, Lee WK, Choi JE, Son JW, Kang YM, et al: A common polymorphism in pre-microRNA-146a is associated with lung cancer risk in a Korean population. Gene 534: 66-71, 2014.

9. Dearth RK, Cui X, Kim H-J, Hadsell DL and Lee AV: Oncogenic transformation by the signaling adaptor proteins insulin receptor substrate (IRS)-1 and IRS-2. Cell Cycle 6: 705-713, 2007.

10. Thomson S, Petti F, Sujka-Kwok I, Epstein D and Haley JD: Kinase switching in mesenchymal-like non-small cell lung cancer lines contributes to EGFR inhibitor resistance through pathway redundancy. Clin Exp Metastasis 25: 843-854, 2008.

11. Chung J-H, Rho JK, Xu X, Lee JS, Yoon HI, Lee CT, Choi YJ, Kim HR, Kim CH and Lee JC: Clinical and molecular evidences of epithelial to mesenchymal transition in acquired resistance to EGFR-TKIs. Lung Cancer 73: 176-182, 2011.

12. Chen J, Han Q and Pei D: EMT and MET as paradigms for cell fate switching. J Mol Cell Biol 4: 66-69, 2012.

13. Hay ED: The mesenchymal cell, its role in the embryo, and the remarkable signaling mechanisms that create it. Dev Dyn 233: 706-720, 2005.

14. Lee JM, Dedhar S, Kalluri R and Thompson EW: The epithelialmesenchymal transition: New insights in signaling, development, and disease. J Cell Biol 172: 973-981, 2006.

15. Thiery JP and Sleeman JP: Complex networks orchestrate epithelial-mesenchymal transitions. Nat Rev Mol Cell Biol 7: 131-142, 2006.

16. Denlinger CE, Ikonomidis JS, Reed CE and Spinale FG: Epithelial to mesenchymal transition: The doorway to metastasis in human lung cancers. J Thorac Cardiovasc Surg 140: 505-513, 2010.

17. Thiery JP: Epithelial-mesenchymal transitions in tumour progression. Nat Rev Cancer 2: 442-454, 2002.

18. Nakata S, Sugio K, Uramoto H, Oyama T, Hanagiri T, Morita M and Yasumoto K: The methylation status and protein expression of CDH1, p16(INK4A), and fragile histidine triad in nonsmall cell lung carcinoma: Epigenetic silencing, clinical features, and prognostic significance. Cancer 106: 2190-2199, 2006.

19. Liu D, Huang C, Kameyama K, Hayashi E, Yamauchi A, Kobayashi S and Yokomise H: E-cadherin expression associated with differentiation and prognosis in patients with non-small cell lung cancer. Ann Thorac Surg 71: 949-954; discussion 954-945, 2001.

20. Hung J-J, Yang M-H, Hsu H-S, Hsu W-H, Liu J-S and Wu K-J: Prognostic significance of hypoxia-inducible factor- $1 \alpha$, TWIST1 and Snail expression in resectable non-small cell lung cancer. Thorax 64: 1082-1089, 2009.

21. Tischler V, Pfeifer M, Hausladen S, Schirmer U, Bonde AK, Kristiansen G, Sos ML, Weder W, Moch H, Altevogt P, et al: L1CAM protein expression is associated with poor prognosis in non-small cell lung cancer. Mol Cancer 10: 127, 2011.

22. Zhuo WL, Wang Y, Zhuo XL, Zhang YS and Chen ZT: Short interfering RNA directed against TWIST, a novel zinc finger transcription factor, increases A549 cell sensitivity to cisplatin via MAPK/mitochondrial pathway. Biochem Biophys Res Commun 369: 1098-1102, 2008.

23. Rho JK, Choi YJ, Lee JK, Ryoo BY, Na II, Yang SH, Kim CH and Lee JC: Epithelial to mesenchymal transition derived from repeated exposure to gefitinib determines the sensitivity to EGFR inhibitors in A549, a non-small cell lung cancer cell line. Lung Cancer 63: 219-226, 2009.

24. Friedman RC, Farh KK-H, Burge CB and Bartel DP: Most mammalian mRNAs are conserved targets of microRNAs. Genome Res 19: 92-105, 2009.

25. Ceppi P and Peter ME: MicroRNAs regulate both epithelial-tomesenchymal transition and cancer stem cells. Oncogene 33: 269-278, 2014.

26. Park S-M, Gaur AB, Lengyel E and Peter ME: The miR-200 family determines the epithelial phenotype of cancer cells by targeting the E-cadherin repressors ZEB1 and ZEB2. Genes Dev 22: 894-907, 2008. 
27. Gregory PA, Bert AG, Paterson EL, Barry SC, Tsykin A, Farshid G, Vadas MA, Khew-Goodall Y and Goodall GJ: The miR-200 family and miR-205 regulate epithelial to mesenchymal transition by targeting ZEB1 and SIP1. Nat Cell Biol 10: 593-601, 2008.

28. Ceppi P, Mudduluru G, Kumarswamy R, Rapa I, Scagliotti GV, Papotti $\mathrm{M}$ and Allgayer $\mathrm{H}$ : Loss of miR-200c expression induces an aggressive, invasive, and chemoresistant phenotype in non-small cell lung cancer. Mol Cancer Res 8: 1207-1216, 2010.

29. Yu T, Li J, Yan M, Liu L, Lin H, Zhao F, Sun L, Zhang Y, Cui Y, Zhang F, et al: MicroRNA-193a-3p and -5p suppress the metastasis of human non-small-cell lung cancer by downregulating the ERBB4/PIK3R3/mTOR/S6K2 signaling pathway. Oncogene 34: 413-423, 2015

30. Moustakas A and Heldin C-H: Signaling networks guiding epithelial-mesenchymal transitions during embryogenesis and cancer progression. Cancer Sci 98: 1512-1520, 2007.

31. Zhou L, Zhao X, Han Y, Lu Y, Shang Y, Liu C, Li T, Jin Z, Fan D and Wu K: Regulation of UHRF1 by miR-146a/b modulates gastric cancer invasion and metastasis. FASEB J 27: 4929-4939, 2013.

32. Karakatsanis A,Papaconstantinou I, Gazouli M,Lyberopoulou A, Polymeneas $\mathrm{G}$ and Voros D: Expression of microRNAs, miR-21, miR-31, miR-122, miR-145, miR-146a, miR-200c, miR-221, miR-222, and miR-223 in patients with hepatocellular carcinoma or intrahepatic cholangiocarcinoma and its prognostic significance. Mol Carcinog 52: 297-303, 2013.

33. Xu B, Wang N, Wang X, Tong N, Shao N, Tao J, Li P, Niu X, Feng N, Zhang L, et al: MiR-146a suppresses tumor growth and progression by targeting EGFR pathway and in a p-ERK-dependent manner in castration-resistant prostate cancer. Prostate 72: 1171-1178, 2012

34. Chen G, Umelo IA, Lv S, Teugels E, Fostier K, Kronenberger P, Dewaele A, Sadones J, Geers C and De Grève J: miR-146a inhibits cell growth, cell migration and induces apoptosis in non-small cell lung cancer cells. PLoS One 8: e60317, 2013.
35. Carew RM, Browne MB, Hickey FB and Brazil DP: Insulin receptor substrate 2 and FoxO3a signalling are involved in E-cadherin expression and transforming growth factor- $\beta 1$ induced repression in kidney epithelial cells. FEBS J 278: 3370-3380, 2011

36. Geng Y, Ju Y, Ren F, Qiu Y, Tomita Y, Tomoeda M, Kishida M, Wang Y, Jin L, Su F, et al: Insulin receptor substrate $1 / 2$ (IRS1/2) regulates $\mathrm{Wnt} / \beta$-catenin signaling through blocking autophagic degradation of dishevelled2. J Biol Chem 289: 11230-11241, 2014.

37. Misra A, Pandey C, Sze SK and Thanabalu T: Hypoxia activated EGFR signaling induces epithelial to mesenchymal transition (EMT). PLoS One 7: e49766, 2012.

38. Cui X, Kim H-J, Kuiatse I, Kim H, Brown PH and Lee AV: Epidermal growth factor induces insulin receptor substrate-2 in breast cancer cells via c-Jun NH(2)-terminal kinase/activator protein-1 signaling to regulate cell migration. Cancer Res 66: 5304-5313, 2006.

39. Dearth RK, Cui X, Kim H-J, Kuiatse I, Lawrence NA, Zhang X, Divisova J, Britton OL, Mohsin S, Allred DC, et al: Mammary tumorigenesis and metastasis caused by overexpression of insulin receptor substrate 1 (IRS-1) or IRS-2. Mol Cell Biol 26: 9302-9314, 2006

40. Chan BT and Lee AV: Insulin receptor substrates (IRSs) and breast tumorigenesis. J Mammary Gland Biol Neoplasia 13: 415-422, 2008.

41. Reuveni H, Flashner-Abramson E, Steiner L, Makedonski K, Song R, Shir A, Herlyn M, Bar-Eli M and Levitzki A: Therapeutic destruction of insulin receptor substrates for cancer treatment. Cancer Res 73: 4383-4394, 2013 